\title{
Recurrent Lumbar Disc Herniation: Results of Revision Surgery and Assessment of Factors that May Affect the Outcome. A Non-Concurrent Prospective Study
}

\author{
Mohammed Ibrahim, Justin Arockiaraj, Rohit Amritanand, Krishnan Venkatesh, Kenny Samuel David \\ Department of Orthopaedics, Spinal Disorders Surgery Unit, Christian Medical College, Vellore, India
}

\begin{abstract}
Study Design: Non-concurrent prospective study.
Purpose: To determine the functional outcome after open 'fragment' discectomy for recurrent lumbar disc herniation, and to analyze the factors that may affect the outcome.

Overview of Literature: Literature search revealed only four studies where the factors affecting the outcome of a revision surgery for recurrent disc herniation have been evaluated. None of these studies analyzed for diabetes, disc degeneration and facet arthropathy. We have analyzed these features, in addition to the demographic and clinical factors.

Methods: Thirty-four patients who underwent the procedure were followed up for an average period of 27.1 months. The Japanese Orthopaedic Association (JOA) score and Oswestry disability index (ODI) were used to assess the functional outcome. Age, gender, smoking, diabetic status, duration of recurrent symptoms, the side of leg pain, level and type of disc herniation, degree of disc degeneration on magnetic resonance imaging, and facet joint arthritis before first and second surgeries, were analyzed as factors affecting the outcome.

Results: The average Hirabayashi improvement in JOA was $56.4 \%$. The mean preoperative ODI was $74.5 \%$ and the mean ODI at final follow-up was $32.2 \%$, the difference being statistically significant $(p<0.01)$. Patients with diabetes, all of whom had poor long term glycemic control, were found to have a poor outcome in terms of ODI improvement $(p=0.03)$.

Conclusions: Open fragment discectomy is a safe and effective surgical technique for the treatment of recurrent disc herniation. However, patients with uncontrolled diabetes may have a less favorable outcome.
\end{abstract}

Keywords: Radiculopathy; Recurrent low back pain; Diabetes mellitus; Discectomy; Outcome assessment

\section{Introduction}

Recurrent disc herniation is indicated when lumbo sacral radiculopathy, caused by ipsilateral or contralateral disc reherniation at the previously operated level, occurs after a pain free interval of at least 6 months, in a patient who had undergone lumbar discectomy [1]. Recurrent disc herniation has been reported in $5 \%$ to $11 \%$ of patients after lumbar discectomy $[2,3]$. Since the overall rate of unsatisfactory results after discectomy is between $5 \%$ to

Received Feb 12, 2015; Revised Mar 31, 2015; Accepted Mar 31, 2015

Corresponding author: Mohammed Ibrahim

Department of Orthopaedics, Spinal Disorders Surgery Unit, Christian Medical College,

Ida Scudder Road, Vellore, Tamilnadu 632004, India

Tel: +91-416-228-2731, Fax: +91-416-223-2035, E-mail: dr.ibrahimortho@gmail.com 
$20 \%[4,5]$, recurrent disc herniation is thus a major cause of surgical failure.

There are a few studies in the literature which have attempted to analyze the risk factors for the development of recurrent disc herniation. However, we could only identify four studies where the factors affecting the outcome of a revision surgery have been evaluated $[1,6-8]$. None of the studies have analyzed diabetes and radiological factors, such as disc degeneration and facet arthropathy. In this study, we undertook to evaluate these features, in addition to the demographic and clinical factors which may affect the outcome after redo fragment discectomy. Identifying such factors would, if possible, help in modifying them to achieve better results, and would assist in explaining prognosis and expected outcome to the patients before surgery.

This study was undertaken to determine the functional outcome after open fragment discectomy for recurrent disc herniation, and to analyze the factors that may affect the final outcome.

\section{Materials and Methods}

This study was a non-concurrent prospective study undertaken at a tertiary care hospital between January 2005 and March 2013. A total of 34 patients who underwent redo fragment discectomy by conventional open method for recurrent disc herniation were followed up for a minimum period of 1 year. Patients with radiological instability were excluded.

The functional outcome was assessed using the Japanese Orthopaedic Association (JOA) score and the Oswestry disability index (ODI) $[9,10]$. The sample size of 34 was calculated from a similar study [6], where JOA was used as an outcome measure. They found an average JOA improvement of $72 \%$. A precision of $15 \%$ was taken for this study, with a $95 \%$ confidence interval.

The data was collected before the surgery and at final follow-up. Age, gender, smoking, presence of diabetes, duration of recurrent symptoms, the side of recurrent leg pain, level and type of disc herniation, degree of disc degeneration on magnetic resonance imaging (MRI), and facet joint arthritis before first and second surgeries were analyzed as factors affecting the outcome.

The improvement in JOA was calculated according to the Hirabayashi score [11] and further classified as done by Takahashi et al. [12], into 4 categories (excellent,
75\%-100\%; good, 50\%-74\%; fair, $25 \%-49 \%$; poor, $<25 \%$ ). The ODI improvement was calculated as the difference between the ODI scores at preoperative and at the final follow-up. The distance a patient is able to walk on a flat surface without support was assessed preoperatively and at follow-up.

The type of disc herniation noted during surgery was classified according to McCulloch and Transfeldt [13]; the degree of disc degeneration seen in MRI at the level of herniation was classified according to Pfirrmann's grading [14]; and the stage of facet joint arthritis was classified according to Fujiwara et al. [15]. The occurrence of complications such as neurological deficits, accidental durotomy, infection and instability were noted. Instability was evaluated by using the radiographic criteria as described by Posner et al. [16].

SPSS ver. 16.0 (SPSS Inc., Chicago, IL, USA) was used to analyze the results. Descriptive statistics were mentioned as mean \pm standard deviation. Student $t$-test was used for analysis of changes in ODI, JOA and walking distance. Mann-Whitney $\mathrm{U}$ and Wilcoxon W tests were used to analyze the effect of factors on the outcome, which were expressed as continuous variables (gender, presence of diabetes, and smoking). Chi-square and Kruskal-Wallis tests were used to analyze the effect of factors on the outcome, which were expressed as categorical variables (age, duration of recurrent symptoms, level and type of disc herniation, the side of pain, disc degeneration and facet joint arthritis in MRI).

\section{Results}

The average follow-up period was 27.1 months (range, 12-82 months). There were 28 male (82.3\%) and 6 female (17.7\%) patients, with a mean age of 45.1 years. Of these, eight patients $(23.5 \%)$ were diabetic and eight patients $(23.5 \%)$ were smokers. The average duration of symptoms before the second surgery was 6.7 months. The most common level of recurrence of herniation was L4-5, which was seen in 20 patients (58.8\%). Ipsilateral recurrent herniation occurred in 23 patients (67.6\%), while 8 patients $(23.5 \%)$ had contralateral herniation. Central herniation was seen in three patients having bilateral leg pain (Table 1).

During the 1st surgery, the most common type of disc degeneration was Pfirrmann's type III, which was seen in 16 patients. At the 2 nd surgery, the degree of disc degen- 
Table 1. Patient data

\begin{tabular}{|c|c|c|c|c|c|c|}
\hline Patient & Age (yr) & Gender & Diabetes & Smoker & Symptom duration (mo) & Side of recurrent pain \\
\hline 1 & 59 & Male & No & No & 2 & Same \\
\hline 2 & 30 & Male & No & No & 5 & Same \\
\hline 3 & 37 & Male & No & No & 2 & Same \\
\hline 4 & 43 & Male & No & No & 2 & Same \\
\hline 5 & 51 & Male & No & No & 1 & Bilateral \\
\hline 6 & 50 & Female & No & No & 1 & Same \\
\hline 7 & 38 & Male & No & No & 1 & Opposite \\
\hline 8 & 43 & Male & No & No & 2 & Same \\
\hline 9 & 34 & Male & No & No & 3 & Same \\
\hline 10 & 32 & Male & No & Yes & 18 & Same \\
\hline 11 & 46 & Male & No & No & 9 & Same \\
\hline 12 & 48 & Male & Yes & Yes & 7 & Opposite \\
\hline 13 & 35 & Male & No & No & 6 & Same \\
\hline 14 & 51 & Female & Yes & No & 7 & Opposite \\
\hline 15 & 55 & Male & No & Yes & 3 & Opposite \\
\hline 16 & 52 & Male & No & No & 6 & Same \\
\hline 17 & 45 & Female & Yes & No & 3 & Same \\
\hline 18 & 36 & Male & No & No & 3 & Same \\
\hline 19 & 46 & Male & No & No & 24 & Opposite \\
\hline 20 & 54 & Female & Yes & No & 3 & Bilateral \\
\hline 21 & 42 & Male & Yes & Yes & 36 & Same \\
\hline 22 & 34 & Female & No & No & 12 & Same \\
\hline 23 & 48 & Female & No & No & 1 & Same \\
\hline 24 & 23 & Male & No & No & 7 & Same \\
\hline 25 & 49 & Male & No & Yes & 2 & Opposite \\
\hline 26 & 71 & Male & Yes & No & 6 & Same \\
\hline 27 & 47 & Male & No & No & 12 & Opposite \\
\hline 28 & 33 & Male & No & No & 12 & Same \\
\hline 29 & 52 & Male & No & Yes & 3 & Same \\
\hline 30 & 71 & Male & Yes & Yes & 10 & Same \\
\hline 31 & 51 & Male & No & No & 6 & Opposite \\
\hline 32 & 47 & Male & No & No & 6 & Same \\
\hline 33 & 60 & Male & Yes & No & 6 & Bilateral \\
\hline 34 & 53 & Male & No & Yes & 4 & Same \\
\hline
\end{tabular}

eration seemed to have progressed, as Pfirrmann's type IV was seen in 18 patients. The progression of disc degeneration was most common in patients who had grade III degeneration before the 1st surgery, and $81 \%$ of patients $(n=13)$ progressed to grade IV at second surgery. Akin to disc degeneration, the facet arthropathy also seemed to have steadily progressed: 19 patients had stage 2 arthropa- thy at 2 nd surgery, as compared to only seven patients who had stage 2 arthropathy before 1st surgery. Two patients had accidental durotomy, which was primarily repaired. There were no other complications. None of the patients had radiological instability at follow-up (Table 2).

The difference in the mean ODI preoperatively (74.5\%) and at final follow-up (32.2\%) was found to be statistically 
Table 2. Patient data

\begin{tabular}{|c|c|c|c|c|c|c|c|}
\hline \multirow[b]{2}{*}{ Patient } & \multirow{2}{*}{$\begin{array}{l}\text { Level of } \\
\text { herniation }\end{array}$} & \multicolumn{2}{|c|}{ Type of disc herniation } & \multicolumn{2}{|c|}{ MRI disc degeneration } & \multicolumn{2}{|c|}{ MRI facet arthropathy } \\
\hline & & $\begin{array}{c}\text { Before 1st } \\
\text { surgery }\end{array}$ & $\begin{array}{l}\text { Before } 2 n d \\
\text { surgery }\end{array}$ & $\begin{array}{l}\text { Before 1st } \\
\text { surgery }\end{array}$ & $\begin{array}{l}\text { Before 2nd } \\
\text { surgery }\end{array}$ & $\begin{array}{c}\text { Before 1st } \\
\text { surgery }\end{array}$ & $\begin{array}{l}\text { Before 2nd } \\
\text { surgery }\end{array}$ \\
\hline 1 & $\llcorner 4-5$ & Extr & Seq & III & IV & 0 & 0 \\
\hline 2 & L3-4, L4-5 & Extr & Seq & $\|$ & III & 0 & 1 \\
\hline 3 & $\llcorner 4-5$ & S. extr & Extr & III & IV & 2 & 2 \\
\hline 4 & L3-4 & Seq & Seq & III & IV & 0 & 0 \\
\hline 5 & $\llcorner 4-5$ & Extr & Extr & $\|$ & $\|$ & 0 & 0 \\
\hline 6 & L5-S1 & Extr & Extr & $\|$ & III & 0 & 1 \\
\hline 7 & L5-S1 & Extr & Extr & $\|$ & $\|$ & 1 & 2 \\
\hline 8 & $\llcorner 4-5$ & S. extr & Extr & III & III & 0 & 0 \\
\hline 9 & L5-S1 & S. extr & Seq & IV & V & 0 & 1 \\
\hline 10 & $\llcorner 4-5$ & Extr & Extr & $\|$ & III & 0 & 0 \\
\hline 11 & $\llcorner 4-5$ & Extr & Extr & III & III & 1 & 1 \\
\hline 12 & $\llcorner 4-5$ & S.extr & S. extr & III & IV & 1 & 2 \\
\hline 13 & L4-5 & Extr & Seq & III & IV & 1 & 1 \\
\hline 14 & L5-S1 & S. extr & Seq & IV & V & 1 & 1 \\
\hline 15 & L4-5 & S. extr & S. extr & III & IV & 2 & 2 \\
\hline 16 & L4-5 & Extr & Seq & $\|$ & IV & 1 & 2 \\
\hline 17 & L5-S1 & Extr & S. extr & III & IV & 1 & 1 \\
\hline 18 & $\llcorner 4-5$ & Extr & Extr & $\|$ & III & 1 & 2 \\
\hline 19 & $\llcorner 4-5$ & Extr & Extr & III & IV & 2 & 3 \\
\hline 20 & L5-S1 & Extr & Extr & $\|$ & IV & 1 & 2 \\
\hline 21 & L5-S1 & S. extr & Extr & IV & IV & 0 & 1 \\
\hline 22 & L4-5 & Extr & Seq & III & IV & 1 & 2 \\
\hline 23 & L5-S1 & Extr & Extr & III & IV & 1 & 2 \\
\hline 24 & L5-S1 & S. extr & Extr & III & III & 1 & 2 \\
\hline 25 & $\llcorner 4-5$ & Extr & Seq & III & IV & 1 & 3 \\
\hline 26 & L3-4 & Extr & Extr & III & IV & 1 & 2 \\
\hline 27 & L4-5 & Extr & Extr & 1 & $\|$ & 2 & 2 \\
\hline 28 & L4-5 & Extr & Extr & $\|$ & III & 2 & 2 \\
\hline 29 & L4-5 & Extr & Extr & $\|$ & IV & 2 & 2 \\
\hline 30 & $\llcorner 4-5$ & Extr & Extr & $\|$ & III & 1 & 2 \\
\hline 31 & L5-S1 & S. extr & S. extr & III & IV & 1 & 2 \\
\hline 32 & L4-5 & Extr & S. extr & $\|$ & IV & 2 & 2 \\
\hline 33 & $\llcorner 4-5$ & Extr & Extr & $\|$ & III & 1 & 2 \\
\hline 34 & L4-5, L5-S1 & Extr & Seq & $\|$ & III & 1 & 2 \\
\hline
\end{tabular}

MRI, magnetic resonance imaging; Extr, trans annular extrusion; Seq, sequestration; S.extr, subannular extrusion.

significant $(p<0.01)$ (Table 3$)$. The average ODI improvement (difference in ODI score at preoperative and at the final follow-up) was $42.6 \%$ (range, $0 \%-78 \%$ ). The mean preoperative JOA was 8.8 and the mean JOA at followup was 20.4; the difference was found to be statistically significant $(p<0.01)$ (Table 1). The average Hirabayashi 
Table 3. Outcome measures

\begin{tabular}{|c|c|c|c|c|c|c|c|}
\hline \multirow{2}{*}{ Patient } & \multicolumn{2}{|c|}{$\begin{array}{c}\text { Japanese Orthopaedic } \\
\text { Association }\end{array}$} & \multicolumn{3}{|c|}{ Oswetry disability index } & \multirow{2}{*}{$\begin{array}{l}\text { Preoperative } \\
\text { walking } \\
\text { distance } \\
\text { (m) }\end{array}$} & \multirow{2}{*}{$\begin{array}{l}\text { Walking } \\
\text { distance at } \\
\text { follow-up } \\
\text { (m) }\end{array}$} \\
\hline & $\begin{array}{l}\text { Before 2nd } \\
\text { surgery }\end{array}$ & $\begin{array}{l}\text { Improvement in } \\
\text { percentage }\end{array}$ & $\begin{array}{l}\text { Before 2nd } \\
\text { surgery }\end{array}$ & Follow-up & Improvement & & \\
\hline 1 & 1 & 64 & 80 & 32 & 48 & 100 & 1,000 \\
\hline 2 & 7 & 68 & 80 & 16 & 64 & 100 & 2,000 \\
\hline 3 & 14 & 86 & 64 & 14 & 50 & 50 & 2,000 \\
\hline 4 & 10 & 73 & 74 & 20 & 54 & 100 & 3,000 \\
\hline 5 & 11 & 83 & 68 & 12 & 56 & 100 & 3,000 \\
\hline 6 & 0 & 39.7 & 94 & 60 & 34 & 50 & 500 \\
\hline 7 & 5 & 91.6 & 86 & 8 & 78 & 50 & 3,000 \\
\hline 8 & 8 & 80 & 56 & 14 & 42 & 300 & 2,000 \\
\hline 9 & 7 & 45 & 72 & 38 & 34 & 100 & 500 \\
\hline 10 & 12 & 41.1 & 76 & 32 & 44 & 100 & 500 \\
\hline 11 & 7 & 40 & 76 & 42 & 34 & 100 & 300 \\
\hline 12 & 7 & 68 & 76 & 18 & 58 & 100 & 2,000 \\
\hline 13 & 9 & 75 & 84 & 28 & 56 & 50 & 500 \\
\hline 14 & 9 & 80 & 64 & 44 & 20 & 100 & 1,000 \\
\hline 15 & 7 & 77 & 72 & 8 & 64 & 500 & 3,000 \\
\hline 16 & 5 & 45 & 90 & 50 & 40 & 50 & 500 \\
\hline 17 & 8 & 61 & 78 & 32 & 46 & 100 & 500 \\
\hline 18 & 7 & 75.5 & 80 & 2 & 78 & 100 & 3,000 \\
\hline 19 & 9 & 75 & 62 & 8 & 54 & 100 & 3,000 \\
\hline 20 & 1 & 53.3 & 86 & 52 & 34 & 50 & 300 \\
\hline 21 & 13 & 25 & 56 & 40 & 16 & 300 & 1,000 \\
\hline 22 & 16 & 70 & 60 & 14 & 46 & 300 & 2,000 \\
\hline 23 & 7 & 81 & 80 & 20 & 60 & 100 & 4,000 \\
\hline 24 & 10 & 79 & 60 & 10 & 50 & 300 & 2,000 \\
\hline 25 & 12 & 64.7 & 86 & 8 & 78 & 50 & 2,000 \\
\hline 26 & 13 & 62.5 & 52 & 14 & 38 & 300 & 2,000 \\
\hline 27 & 12 & 58 & 86 & 26 & 60 & 50 & 1,000 \\
\hline 28 & 12 & 11 & 88 & 70 & 18 & 50 & 100 \\
\hline 29 & 14 & 40 & 78 & 42 & 36 & 100 & 300 \\
\hline 30 & 3 & 0 & 90 & 94 & -4 & 100 & 100 \\
\hline 31 & 14 & 0 & 70 & 70 & 0 & 100 & 100 \\
\hline 32 & 8 & 57 & 80 & 82 & -2 & 100 & 50 \\
\hline 33 & 10 & 10.5 & 69 & 58 & 11 & 100 & 400 \\
\hline 34 & 11 & 38 & 62 & 18 & 44 & 100 & 300 \\
\hline
\end{tabular}

improvement percentage in JOA at follow-up was $56.4 \%$ (range, 0\%-91.6\%). According to JOA improvement, 11 patients $(32.4 \%)$ patients had excellent outcome, 11 patients $(32.4 \%)$ had good outcome, 8 patients $(23.5 \%)$ patients had fair outcome, and four patients (11.8\%) had poor outcome after the 2 nd surgery (Table 3 ). The mean 
preoperative walking distance was 127.9 meters, whereas at final follow-up it was $1,380.8$ meters. The average improvement in walking distance was found to be 1,252 meters, which was statistically significant $(p<0.01)$.

Among the clinical factors evaluated, patients with diabetes, all of whom had a poor long term glycemic control (HBA1c>7.5), were found to have a poor outcome in terms of ODI improvement, which was statistically significant ( $p=0.03)$. Age, gender, smoking, duration of recurrent symptoms, the side of leg pain, level and type of disc herniation, degree of disc degeneration and facet joint arthritis before 1st and 2nd surgeries were not found to affect the outcome ( $p>0.05)$.

Of the 34 patients, five patients (14.7\%) complained of significant axial back pain and leg pain at follow-up (average 32.8 months after the 2 nd surgery). None of them responded to non-operative treatment given for 6-8 weeks, from the time they presented with these complaints (first follow-up). All patients had to undergo a 3rd surgery, in the form of fusion (instrumented PLIF), after which they were followed-up for an average of 42.2 months (range, 10-70 months). Average ODI improvement was 59.6, with three patients having minimal disability and two with moderate disability. The average JOA improvement was $68.6 \%$. With the Takahashi et al. [12] classification of JOA improvement, four patients $(80 \%)$ had satisfactory outcome after the 3 rd surgery. The fusion was assessed using the modified Lee's criteria [17]. Accordingly, two patients had definitive fusion, two had probable fusion and one patient had possible pseudoarthrosis.

\section{Discussion}

Recurrent lumbar disc herniation is a major cause of postoperative failure in patients who undergo lumbar discectomy. The duration after the first surgery that should elapse before a patient is said to have recurrent herniation is not clearly defined in literature. Some studies have considered 3 months [6], while most others consider 6 months as the time interval. Although in the clinical settings no strict time interval is required to elapse before the diagnosis of a recurrent disc herniation can be made, Lee et al. [18] have noted in their review article that before 6 months have elapsed, it is difficult to distinguish a recurrent disc with postoperative changes and scar tissue, in an MRI. There are studies showing possible distinction between scar and recurrent herniation with contrast enhanced MRI scans, but there is no study distinguishing postoperative changes, scar tissue and recurrent herniation in MRI within 6 months of surgery. Hence, it is difficult to objectively diagnose a recurrent disc herniation as the cause of patient's symptoms within 6 months of surgery. This study included a uniform cohort of patients, with a minimum of 6 months pain free interval after the first discectomy, who were treated with redo open 'fragment' discectomy.

The treatment of recurrent disc herniation is varied, with some authors advocating fusion while many others prefer repeat discectomy. Studies have indicated the outcome of repeat discectomy to be similar to both primary discectomy [1,19-21] and also to fusion [22,23]; therefore, repeat discectomy seems to be a better choice in patients without instability.

In this study, the functional outcome was assessed using the ODI and Hirabayashi percentage improvement of JOA, and its classification used by Takahashi et al. [12]. Tsai et al. [7] and Guo et al. [8] in their retrospective studies, have used the Macnab's criteria which is a truly subjective scale and does not take into account objective factors like walking distance, presence of motor or sensory deficits, bladder dysfunction, and other subjective factors involving activities of daily living like washing, lifting, sitting and standing. In these aspects the JOA seems to be a better measurement tool which incorporates both objective as well as subjective data. Suk et al. [1], in their retrospective evaluation of 28 patients with recurrent disc herniation, have used the VAS score for 'radiating leg pain' as the only outcome measure; the suitability of this outcome measure (VAS score) can be questioned, as many other factors like back pain, neurological deficits etc., can also affect the final outcome.

In our present study, at follow-up, the average Hirabayashi improvement percentage in JOA was $56.4 \%$ (range, 0\%-91.6\%). According to improvement in JOA, $64.8 \%(n=22)$ had a satisfactory outcome. The average ODI improvement was $42.6 \%$ (range, $0 \%-78 \%$ ). At follow-up, 15 patients (44.2\%) had minimal disability. The results were comparable to other studies $[8,21]$ where JOA and ODI were used as outcome measures.

Assessment of factors affecting the outcome of recurrent disc herniation helps to prognosticate the outcome in a patient who has already had a 'failed back surgery'. Four studies $[1,6-8]$ could be found in literature, which have tried to analyze the factors affecting the outcome after a 
redo discectomy. As in our study, in these studies also, age, gender, smoking, level of herniation, type of herniation, side of recurrence and duration of recurrent symptoms were not found to affect the final outcome.

Patients with diabetes have been found to have a poor outcome after lumbar discectomy [24,25], decompression [24-27], and fusion $[26,28,29]$ surgeries. In our study, all patients with diabetes had Type II diabetes, were on insulin therapy, but with poor long term glycemic control (HBA1c $>7.5$ in all patients preoperatively and at final follow-up). According to the World Health Organization clinical classification of diabetes, all the 8 diabetic patients were classified as stage II (insulin requiring for control) [30], and were found to have a poor outcome in terms of improvement in ODI $(p=0.03)$. In their study of outcome of decompression in diabetic patients with lumbar canal stenosis, Cinotti et al. [26] found $80 \%$ of the diabetic patients who underwent decompression had delayed nerve conduction velocity in lower limbs as compared to $25 \%$ among the non-diabetics. As noted by Simpson et al. [24], the poor results in the diabetic patients may be related to coexisting diabetic neuropathy, associated microvascular disease that affects the spinal nerve roots, or to the failure of the nerve roots of these patients to recover after decompressive procedures. The presence of diabetes per se may not contribute to a poor outcome, but the presence of uncontrolled diabetes with its complications like neuropathy, may result in a less favorable outcome. In this study, we did not evaluate nerve conduction or vascular insufficiency in lower limbs, as a routine in any of the patients. Hence, we could not identify the reason why diabetic patients had a poor outcome when compared to patients without diabetes. Although we found that patients having diabetes with poor long term glycemic control had a less favorable outcome, we did not have a control group which could have enabled us to compare these patients with the individuals who may have well controlled diabetes. This is a limitation of our study, and an area which needs further research.

With the evolution of motion preserving surgeries in the lumbar spine, we wanted to assess the effect of structures that allow motion at a given spinal segment i.e., the intervertebral disc and the facet joints. We found that the extent of disc degeneration observed in MRI before 1st and 2nd surgery affected the outcome in terms of ODI and its subsequent improvement at follow-up, with a borderline statistical significance ( $p=0.07$ and $p=0.08$ ). The degree of facet joint arthritis before 1st and 2nd surgery did not affect the final outcome.

In the only prospective study evaluating 26 patients undergoing repeat discectomies for ipsilateral recurrent disc herniation, and considering a similar group of 50 patients who underwent primary discectomies as controls, Cinotti et al. [19] found that the patients in their study group had significantly more severe disc degeneration as compared with the control group. Although in our study we could not ascertain the degree of disc degeneration to be a risk factor for recurrent herniation (we did not have a control group), patients showing more severe disc degeneration in MRI at first surgery were found to be more symptomatic before second surgery in terms of ODI. The marginal $p$-values we derived with respect to the effect of disc degeneration on the outcome, might be because of the small sample size. The sample size though small, is comparable to other such studies in literature.

\section{Conclusions}

With this study, we can conclude that open fragment discectomy is a safe and effective surgical technique for the treatment of recurrent lumbar disc herniation. Patients with diabetes and poor long term glycemic control may have a less favorable outcome, though further research in this regard is needed to ascertain what complications of diabetes contribute to poor outcome.

\section{Conflict of Interest}

No potential conflict of interest relevant to this article was reported.

\section{Acknowledgments}

We would like to thank Mrs. Grace Rebekah, Clinical Epidemiological Unit, Christian Medical College, Vellore, for statistical analysis of data.

\section{References}

1. Suk KS, Lee HM, Moon SH, Kim NH. Recurrent lumbar disc herniation: results of operative management. Spine (Phila Pa 1976) 2001;26:672-6.

2. Connolly ES. Surgery for recurrent lumbar disc herniation. Clin Neurosurg 1992;39:211-6. 
3. Fandino J, Botana C, Viladrich A, Gomez-Bueno J. Reoperation after lumbar disc surgery: results in 130 cases. Acta Neurochir (Wien) 1993;122:102-4.

4. Greenwood J Jr, McGuire TH, Kimbell F. A study of the causes of failure in the herniated intervertebral disc operation: an analysis of sixty-seven reoperated cases. J Neurosurg 1952;9:15-20.

5. Pheasant H. Sources of failure in laminectomies. Orthop Clin North Am 1975;6:319-29.

6. Dai LY, Zhou Q, Yao WF, Shen L. Recurrent lumbar disc herniation after discectomy: outcome of repeat discectomy. Surg Neurol 2005;64:226-31.

7. Tsai CH, Hsu HC, Chen YJ, Lin CJ, Chen HT. Recurrent lumbar disc herniation after discectomy: clinical result of repeated discectomy and analysis of factors affecting surgical outcome. Mid Taiwan J Med 2007;12:125-32.

8. Guo JJ, Yang H, Tang T. Long-term outcomes of the revision open lumbar discectomy by fenestration: a follow-up study of more than 10 years. Int Orthop 2009;33:1341-5.

9. Fairbank JC, Couper J, Davies JB, O’Brien JP. The Oswestry low back pain disability questionnaire. Physiotherapy 1980;66:271-3.

10. Fairbank JC, Pynsent PB. The Oswestry Disability Index. Spine (Phila Pa 1976) 2000;25:2940-52.

11. Hirabayashi K, Miyakawa J, Satomi K, Maruyama T, Wakano K. Operative results and postoperative progression of ossification among patients with ossification of cervical posterior longitudinal ligament. Spine (Phila Pa 1976) 1981;6:354-64.

12. Takahashi S, Suzuki A, Toyoda H, et al. Characteristics of diabetes associated with poor improvements in clinical outcomes after lumbar spine surgery. Spine (Phila Pa 1976) 2013;38:516-22.

13. McCulloch JA, Transfeldt EE. Macnab's backache. 3rd ed. Baltimore, MD: Williams and Wilkins; 1977.

14. Pfirrmann CW, Metzdorf A, Zanetti M, Hodler J, Boos N. Magnetic resonance classification of lumbar intervertebral disc degeneration. Spine (Phila Pa 1976) 2001;26:1873-8.

15. Fujiwara A, Tamai K, Yamato $M$, et al. The relationship between facet joint osteoarthritis and disc degeneration of the lumbar spine: an MRI study. Eur Spine J 1999;8:396-401.

16. Posner I, White AA 3rd, Edwards WT, Hayes WC. A biomechanical analysis of the clinical stability of the lumbar and lumbosacral spine. Spine (Phila Pa 1976) 1982;7:374-89.

17. Lee CK, Vessa P, Lee JK. Chronic disabling low back pain syndrome caused by internal disc derangements: the results of disc excision and posterior lumbar interbody fusion. Spine (Phila Pa 1976) 1995;20:35661.

18. Lee JK, Amorosa L, Cho SK, Weidenbaum M, Kim Y. Recurrent lumbar disk herniation. J Am Acad Orthop Surg 2010;18:327-37.

19. Cinotti G, Gumina S, Giannicola G, Postacchini F. Contralateral recurrent lumbar disc herniation: results of discectomy compared with those in primary herniation. Spine (Phila Pa 1976) 1999;24:800-6.

20. Papadopoulos EC, Girardi FP, Sandhu HS, et al. Outcome of revision discectomies following recurrent lumbar disc herniation. Spine (Phila Pa 1976) 2006;31:1473-6.

21. Patel MS, Braybrooke J, Newey M, Sell P. A comparative study of the outcomes of primary and revision lumbar discectomy surgery. Bone Joint J 2013;95:90-4.

22. El Shazly AA, El Wardany MA, Morsi AM. Recurrent lumbar disc herniation: a prospective comparative study of three surgical management procedures. Asian J Neurosurg 2013;8:139-46.

23. Fu TS, Lai PL, Tsai TT, Niu CC, Chen LH, Chen WJ. Long-term results of disc excision for recurrent lumbar disc herniation with or without posterolateral fusion. Spine (Phila Pa 1976) 2005;30:2830-4

24. Simpson JM, Silveri CP, Balderston RA, Simeone FA, An HS. The results of operations on the lumbar spine in patients who have diabetes mellitus. J Bone Joint Surg Am 1993;75:1823-9.

25. Freedman MK, Hilibrand AS, Blood EA, et al. The impact of diabetes on the outcomes of surgical and nonsurgical treatment of patients in the spine patient outcomes research trial. Spine (Phila Pa 1976) 2011;36:290-307.

26. Cinotti G, Postacchini F, Weinstein JN. Lumbar spinal stenosis and diabetes: outcome of surgical decompression. J Bone Joint Surg Br 1994;76:215-9.

27. Arinzon Z, Adunsky A, Fidelman Z, Gepstein R. Outcomes of decompression surgery for lumbar spinal stenosis in elderly diabetic patients. Eur Spine J 2004;13:32-7.

28. Browne JA, Cook C, Pietrobon R, Bethel MA, Richardson WJ. Diabetes and early postoperative 
outcomes following lumbar fusion. Spine (Phila Pa 1976) 2007;32:2214-9.

29. Glassman SD, Alegre G, Carreon L, Dimar JR, Johnson JR. Perioperative complications of lumbar instrumentation and fusion in patients with diabetes mellitus. Spine J 2003;3:496-501.
30. Alberti KG, Zimmet PZ. Definition, diagnosis and classification of diabetes mellitus and its complications. Part 1: diagnosis and classification of diabetes mellitus provisional report of a WHO consultation. Diabet Med 1998;15:539-53. 